\title{
USE OF THE EPIPHYTIC DIATOMS TO ESTIMATE THE ECOLOGICAL STATUS OF LAKE MOGAN
}

\author{
SANAL, $\mathrm{M}^{{ }^{*}}{ }^{*}-$ DEMIR, $\mathrm{N}^{2}$ \\ ${ }^{1}$ Republic of Turkey, Ministry of Culture and Tourism, Directorate General of Investments and \\ Enterprises, 06100 Ankara, Turkey \\ ${ }^{2}$ Ankara University, Faculty of Agriculture, Department of Fisheries and Aquaculture \\ Engineering, 06110 Ankara, Turkey \\ (phone:+90-312-596-1643; fax:+90-312-318-5298) \\ *Corresponding author \\ e-mail: m.sanal06@hotmail.com; phone: +90-312-470-7358; fax:+90-312-223-5448 \\ (Received 19 ${ }^{\text {th }}$ Mar 2018; accepted 30 ${ }^{\text {th }}$ May 2018)
}

\begin{abstract}
In this study, the seasonal succession of the diatoms on Phragmites australis community was investigated in Lake Mogan (Turkey), and the epiphytic diatom community was used to estimate the ecological condition of the lake. Five stations were chosen in the littoral region of the lake, and diatom samples were taken from the $P$. australis monthly between January and November 2013. Two stations were selected in the pelagial region and chlorophyll $a$ concentrations were determined. Lake water temperature, $\mathrm{pH}$, dissolved oxygen concentration, conductivity and Secchi depths were measured, and total nitrogen, total phosphorus and silicate were analyzed. A total of 61 epiphytic diatom species were identified in Lake Mogan. The dominant species were those with tolerance to $\alpha-\beta$ mesosaprobic conditions. According to the mean Secchi depth, chlorophyll and total phosphorus concentrations, the lake was eutrophic. The IDG, IPS, DESCY, WAT, IDP and TDI indices, calculated according to epiphytic diatoms, indicated an ecological quality between medium and poor (classes III-IV). Among them, IDG and IPS are recommended for monitoring of ecological status in the lake because these indices also integrated all diatom taxa within the samples. The occurrence of pollution-tolerant species and the fact that most indices showed an ecological quality of medium or poor indicated an increase of organic matter and eutrophication in Lake Mogan.
\end{abstract}

Keywords: epiphyton, reed, shallow lake, water quality, chlorophyll

\section{Introduction}

The most widespread algae, diatoms, can be found in all water resources, from small streams to sea, and from clean to polluted water. Diatoms include many different forms and can be found on all types of substrates; and they can easily be sampled in great numbers from small surfaces in aqueous environments. They can also be sampled from artificial surfaces, so that their rapid response to changes in water quality, together with a fast access to results by means of diatom indices, make them advantageous for use in monitoring water quality. Among their disadvantages are the need for taxonomic knowledge in identifying them, and the difficulty in separating dead and live cells. Diatoms have been used as an indicator of water quality since 1949, and for this reason many species have been classified according to their tolerance and sensitivity to pollution. The cells do not break up easily because they contain silica, and are indicators of long term as well as short term changes (Cox, 1991; Round, 1991; Smol and Stoermer, 2010). Diatoms are particularly used as indicators of $\mathrm{pH}$, conductivity, salinity and trophic level (Cox, 1991). 
The Water Framework Directive, adopted by and implemented in the European Union in the year 2000, classifies water resources by their ecological qualities and aims to bring water to a good ecological quality. Phytobenthos, according to Water Framework Directive, is one of the indicators used to determine the ecological quality of standing and flowing waters (Anonymous, 2000). The use of epiphytic diatoms, which are a component of lake phytobenthos, is important to estimate the ecological quality of shallow lakes.

Because diatoms form a large proportion (90-95\%) of benthic habitats of water ecosystems, they have become an important part of water quality monitoring studies (Ács et al., 2004). The occurrence of a particular diatom species in different bodies of water, from clean to polluted waters, enables the use of indices of diatom variety. Diatom indices are based on tolerance and/or sensivity of taxa (genus or species) and allow the assessment of water quality using the structure of diatom communities.

Although there have been many studies of the use of diatoms in determining water quality and/or ecological quality in flowing water (Atıc1, 1997; Eloranta and Soinien, 2002; Blanco et al., 2004; Johnson et al., 2006; Kalyoncu et al., 2009; Salomoni et al., 2011; Gün, 2011; Solak and Ács, 2011; Kivrak et al., 2012; Tokatl1, 2012; Kalyoncu and Şerbetçi, 2013; Taş et al., 2015; Demir et al., 2017; Toudjani et al., 2017;), a limited number of such studies have been conducted on lakes (Poulíčková et al., 2004; Ács et al., 2005; Kovács et al., 2007; Lirika et al., 2013; Tokatl1, 2013; Kavya and Ulavi, 2014; Szabo et al., 2017). For this reason, studies of the use of diatoms to determine ecological quality in lakes are of great importance. Tokatlı (2013) reported that the TDI index had been calculated in Porsuk Reservoir using epipelic diatoms, and that according to this index it was mesotrophic.

Several studies have been conducted in limnology, water quality or phytoplankton in Lake Mogan (Akbulut and Akbulut, 2002; Fakığlu and Pulatsü, 2005; Atıcı et al., 2010; Yerli et al., 2012; Atıcı and Tokatlı, 2014; Şanal et al., 2015). Demir et al., (2014) researched the possibility of using Q index in assessing the ecological quality class of Lake Mogan. Estimates of the ecological quality made in connection with phytoplankton community structure in the lake were compared with the nutrient status. The results showed that $\mathrm{Q}$ index could be used in monitoring ecological quality in Lake Mogan. It is a shallow lake surrounded by a belt of reed Phragmites australis (Cavanilles) Trinius et Steudel on its shores. According to King et al., (2006), P. australis is recommended as an ideal plant for sampling epiphytic diatoms when determining ecological quality in lakes.

The aim of the present study was to determine the ecological quality of Lake Mogan, which is an important example of a shallow lake under anthropogenic pressure, using epiphytic diatoms found on Phragmites australis, and to determine the diatom composition in the lake.

\section{Materials and Methods}

\section{Study Site}

Lake Mogan is situated in Sakarya Basin, $20 \mathrm{~km}$ south of Ankara in Turkey, between

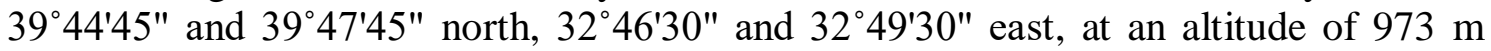
above sea level. It has an area of $5.7 \mathrm{~km}^{2}$, and an average depth of $2.4 \mathrm{~m}$. The maximum depth is $4.25 \mathrm{~m}$. The lake is $5.4 \mathrm{~km}$ long and approximately $1.7 \mathrm{~km}$ wide. Its shore length is approximately $16 \mathrm{~km}$ (Demir et al., 2014). 
Because they have ecological and recreational importance, the wetland-marsh areas around Lakes Mogan and Eymir have been designated as the Gölbaşı Special Environmental Protection Area (Anonymous, 2015). Lake has been heavily influenced by human actions ranging from agricultural and domestic to semi-industrial pollution sources. The lake under the pression of anthropogenic pollution due to the external phosphorus load resulting from detergents in waste water effluents and fertilizers in agricultural run-off from nearby areas (Fakığlu and Pulatsü, 2005; Topçu and Pulatsü, 2017).

\section{Analysis}

In this study, samples were taken once a month from Lake Mogan between January and November 2013. Samples of water and epiphytic diatoms were taken at five stations chosen in the littoral region of the lake (stations 1,2,3,4 and 5), and other water samples were taken at two stations in the pelagial region (stations 7 selected at the deepest point of the lake and station 6 close to the entry) in order to determine the concentration of chlorophyll a (Figure 1). In December, samples could not be taken because the lake was frozen.

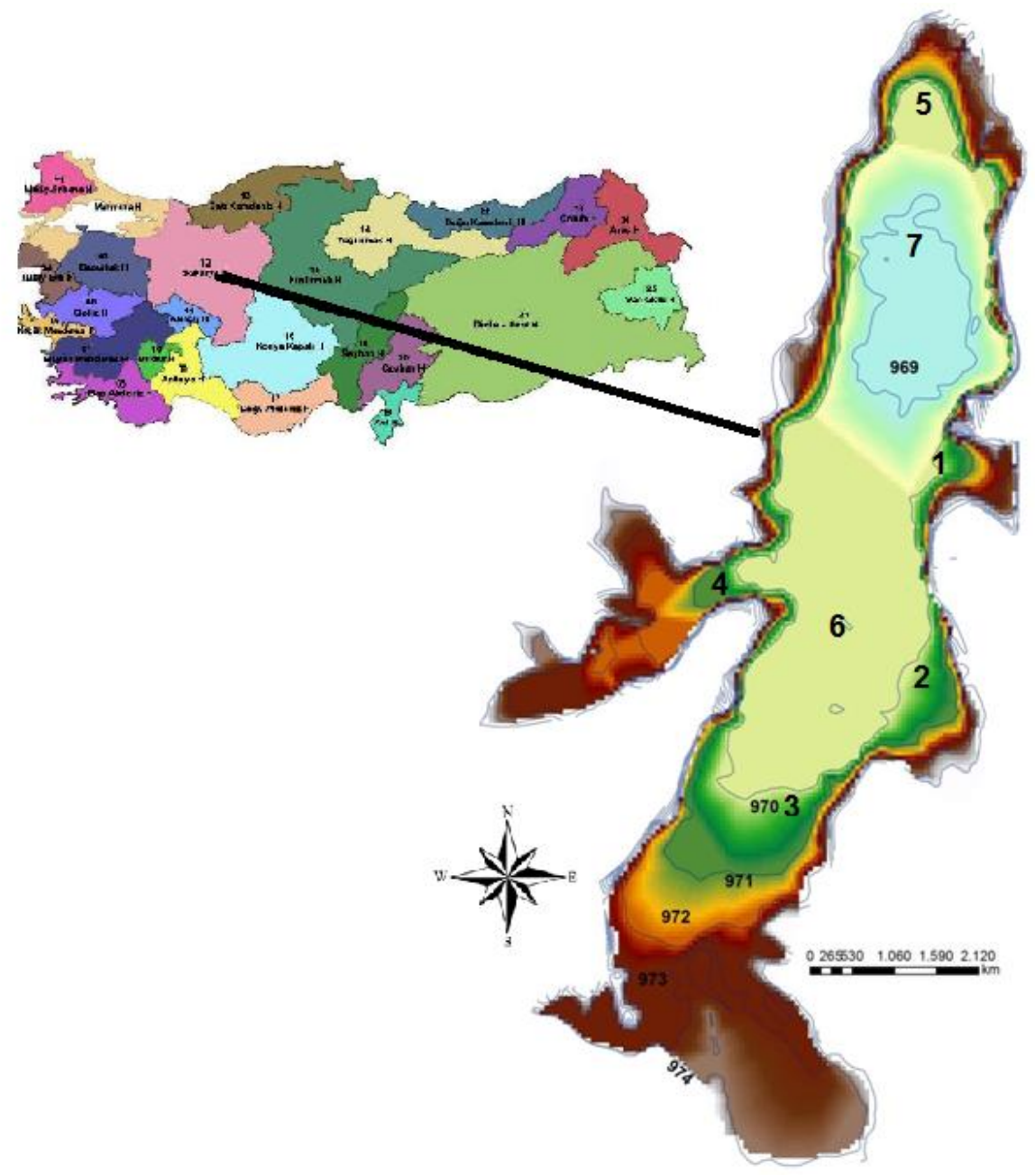

Figure 1. Sakarya basin and sampling stations in Lake Mogan (1-5:littoral stations, 6- 7: pelagial stations) 
Two independent parallel water samples were collected from below the lake surface with a 21 Ruttner water sampler. At each station in the littoral region, five reeds (Phragmites australis (Cavanilles) Trinius et Steudel) were collected, and 20-cm sections were taken from below the water surface (King et al., 2006). The sections of reeds were placed in a container and agitated with $70 \%$ ethanol to separate the epiphytic algae from the plants and to bring them to a homogeneous state (TS EN 15708, 2010). The diatoms were treated with $\mathrm{H}_{2} \mathrm{O}_{2}$ to remove organic material, and triplicate stable preparations were made using Naphrax (CEN, 2004).

Identification of the diatoms was performed by measuring them with an imaging system using a Leica DFC295 camera and the LAS vs 4.8 program, and referring to the relevant taxonomic literature (Krammer and Lange-Bertalot, 1985, 1986, 1988, 1991a, 1991b; Cox, 1996; Lange-Bertalot, 2013). Also, all of the taxonomic data was updated in accordance with algae databases (Guiry and Guiry, 2018). Triplicate preparats were counted for each station. At least 400 cells were counted in each preparat, and SLA, DESCY, IDSE, SHE, TDI, CEE, IPS, IBD, IDAP, EPI-D, DI-CH, IDP, SID, TID, WAT, \%PT, IDG, LOBO and TDIL diatom indices were calculated using the OMNIDIA vs 6.06 package (Lecointe et al., 1993). Most of the indices are modified from the weighted average equation of Zelinka and Marvan's index (Bellinger and Sigee, 2010). In addition, ecological characteristics were determined according to Denys (1991), Håkansson (1993), Hofmann (1994) and Van Dam et al., (1994). In the chlorophyll $a$ analysis, spectrophotometric method was used. Dublicate water samples of $500 \mathrm{ml}$ taken from below the surface at two stations were filtered with Whatman GF/C filter paper and extracted with acetone, after which the optical density of the extract was read on a spectrophotometer (Shimadzu UV 1240) at wavelengths of 630, 645 and $665 \mathrm{~nm}$ (Strickland and Parsons, 1972; APHA, 1998).

Measurements were made at the stations of Secchi depth using a $20 \mathrm{~cm}$ diameter Secchi disc, of dissolved oxygen, $\mathrm{pH}$ and electrical conductivity, using a YSI ProPlus Multiparameter. Total phosphorus, total nitrogen and silica were analyzed by standard methods (APHA, 1998).

\section{Statistical analyses}

The monthly and station-to-station variations in water temperature, Secchi depth, dissolved oxygen, $\mathrm{pH}$, electrical conductivity, total phosphorus, total nitrogen and silicate values in Lake Mogan were analysed using a one-way variance analysis (ANOVA). In addition, the correlations between diatom indices and physico-chemical parameters were examined with Spearman correlation analysis using SPSS vs17 package. All statistical calculations and checks were conducted according to Kesici and Kocabaş (2007).

\section{Results}

The monthly variations in water temperature, Secchi depth, dissolved oxygen, $\mathrm{pH}$, $\mathrm{EC}$, total nitrogen and silicate in the lake were found to be significant $(\mathrm{p}<0.05)$, but the variations in these parameters between stations were found not to have statistical significance. An evaluation was made according to the average of the results of the monthly measurements in the lake and according to the Surface Water Quality Directive and trophic status criteria (Anonymous, 2016) (Table 1). 
Table 1. Monthly values of physical and chemical water quality parameters in Lake Mogan between January and November 2013 (mean \pm standard error) ${ }^{I}$

\begin{tabular}{|c|c|c|c|c|c|c|c|c|c|c|c|c|c|c|c|c|}
\hline Month & $\underset{\mathrm{n}=14^{2}}{\mathbf{J}}$ & $\begin{array}{c}\mathbf{F} \\
\mathrm{n}=14\end{array}$ & $\underset{\mathrm{n}=14}{\mathbf{M}}$ & $\underset{\mathrm{n}=14}{\mathbf{A}}$ & $\underset{\mathrm{n}=14}{\mathbf{M}}$ & $\underset{n=14}{\mathbf{J}}$ & $\underset{n=14}{\mathbf{J}}$ & $\underset{\mathrm{n}=14}{\mathbf{A}}$ & $\underset{n=14}{S}$ & $\underset{n=14}{\mathbf{O}}$ & $\begin{array}{c}\mathbf{N} \\
\mathrm{n}=14\end{array}$ & $\begin{array}{c}\operatorname{Mean} \pm \\
\mathbf{S E} \\
n=154\end{array}$ & $\begin{array}{c}\text { WQ } \\
\text { I }\end{array}$ & $\begin{array}{c}\text { WQ } \\
\text { II }\end{array}$ & $\begin{array}{c}\text { WQ } \\
\text { III }\end{array}$ & $\begin{array}{l}\text { WQ } \\
\text { IV }\end{array}$ \\
\hline$\underset{{ }^{\circ} \mathrm{C}}{\text { Temp. }}$ & $\begin{array}{c}4.83 \\
\pm 0,17\end{array}$ & $\begin{array}{l}10.22 \\
\pm 0.23\end{array}$ & $\begin{array}{c}9.09 \\
\pm 0.13\end{array}$ & $\begin{array}{l}21.61 \\
\pm 0.43\end{array}$ & $\begin{array}{l}23.75 \\
\pm 0.39\end{array}$ & $\begin{array}{l}25.49 \\
\pm 0.20\end{array}$ & $\begin{array}{l}25.54 \\
\pm 0.27\end{array}$ & $\begin{array}{l}25.19 \\
\pm 0.29\end{array}$ & $\begin{array}{l}18.23 \\
\pm 0.22\end{array}$ & $\begin{array}{l}13.84 \\
\pm 0.31\end{array}$ & $\begin{array}{c}7.41 \\
\pm 0.28\end{array}$ & $\begin{array}{l}16.84 \\
\pm 8.01 \\
\end{array}$ & - & - & - & - \\
\hline pH & $\begin{array}{c}8.20 \\
\pm 0.02\end{array}$ & $\begin{array}{c}8.01 \\
\pm 0.05\end{array}$ & $\begin{array}{c}8.44 \\
\pm 0.05\end{array}$ & $\begin{array}{c}8.47 \\
\pm 0.02\end{array}$ & $\begin{array}{c}9.62 \\
\pm 0.01\end{array}$ & $\begin{array}{c}9.49 \\
\pm 0.03\end{array}$ & $\begin{array}{c}9.85 \\
\pm 0.02\end{array}$ & $\begin{array}{c}9.78 \\
\pm 0.04\end{array}$ & $\begin{array}{l}10.96 \\
\pm 0.22 \\
\end{array}$ & $\begin{array}{l}10.87 \\
\pm 0.03\end{array}$ & $\begin{array}{l}10.94 \\
\pm 0.14\end{array}$ & $\begin{array}{c}9.51 \\
\pm 1.11 \\
\end{array}$ & $6-9$ & $6-9$ & $6-9$ & $6-9$ \\
\hline $\begin{array}{c}\mathrm{EC} \\
\mu \mathrm{S} / \mathrm{cm}\end{array}$ & $\begin{array}{c}1262.5 \\
\pm 20.6\end{array}$ & $\begin{array}{c}1265 \\
\pm 10.2\end{array}$ & $\begin{array}{c}1371.5 \\
\pm 16.0\end{array}$ & $\begin{array}{c}1300.1 \\
\pm 29.9\end{array}$ & $\begin{array}{c}1364.3 \\
\pm 14.3 \\
\end{array}$ & $\begin{array}{c}1284.1 \\
\pm 42.5\end{array}$ & $\begin{array}{c}1256.2 \\
\pm 28.2\end{array}$ & $\begin{array}{c}1280.2 \\
\pm 23.3\end{array}$ & $\begin{array}{c}1267.4 \\
\pm 12.9\end{array}$ & $\begin{array}{c}1291.0 \\
\pm 13.1\end{array}$ & $\begin{array}{c}1361.7 \\
\pm 16.7\end{array}$ & $\begin{array}{c}1300 \\
\pm 45\end{array}$ & $<400$ & 1000 & 3000 & $>3000$ \\
\hline $\begin{array}{c}\mathrm{DO} \\
\mathrm{mgO}_{2} / \mathrm{l}\end{array}$ & $\begin{array}{c}8.66 \\
\pm 0.56 \\
\end{array}$ & $\begin{array}{c}8.51 \\
\pm 0.20 \\
\end{array}$ & $\begin{array}{c}7.39 \\
\pm 0.30 \\
\end{array}$ & $\begin{array}{r}8.33 \\
\pm 0.15 \\
\end{array}$ & $\begin{array}{c}6.52 \\
\pm 0.25 \\
\end{array}$ & $\begin{array}{c}6.48 \\
\pm 0.25 \\
\end{array}$ & $\begin{array}{c}5.91 \\
\pm 0.86 \\
\end{array}$ & $\begin{array}{c}7.94 \\
\pm 0.30 \\
\end{array}$ & $\begin{array}{c}8.39 \\
\pm 0.17 \\
\end{array}$ & $\begin{array}{l}10.22 \\
\pm 0.38 \\
\end{array}$ & $\begin{array}{l}10.95 \\
\pm 0.62 \\
\end{array}$ & $\begin{array}{r}8.12 \\
\pm 1.60 \\
\end{array}$ & $>8$ & 6 & 3 & $<3$ \\
\hline $\begin{array}{c}\begin{array}{c}\text { Silicate } \\
\mathrm{mg} / \mathrm{l}\end{array} \\
\end{array}$ & $\begin{array}{c}7.60 \\
\pm 2.07\end{array}$ & $\begin{array}{c}4.21 \\
\pm 1.22 \\
\end{array}$ & $\begin{array}{c}3.14 \\
\pm 0.98\end{array}$ & $\begin{array}{c}5.00 \\
\pm 0.57\end{array}$ & $\begin{array}{c}5.36 \\
\pm 1.02\end{array}$ & $\begin{array}{c}8.71 \\
\pm 1.52 \\
\end{array}$ & $\begin{array}{c}6.29 \\
\pm 1.18\end{array}$ & $\begin{array}{c}7.93 \\
\pm 0.67\end{array}$ & $\begin{array}{c}8.43 \\
\pm 1.13 \\
\end{array}$ & $\begin{array}{c}8.43 \\
\pm 1.20 \\
\end{array}$ & $\begin{array}{c}8.43 \\
\pm 2.22 \\
\end{array}$ & $\begin{array}{c}6.68 \\
\pm 1.91 \\
\end{array}$ & $\begin{array}{l}\text { Oligo- } \\
\text { trophic }\end{array}$ & $\begin{array}{l}\text { Meso- } \\
\text { trophic }\end{array}$ & $\begin{array}{c}\text { Eu- } \\
\text { trophic }\end{array}$ & $\begin{array}{l}\text { Hyper- } \\
\text { trophic }\end{array}$ \\
\hline $\begin{array}{c}\mathbf{T P} \\
\mu \mathrm{g} / \mathbf{l}\end{array}$ & $\begin{array}{r}82.00 \\
\pm 50.00 \\
\end{array}$ & $\begin{array}{r}84.29 \\
\pm 32.07 \\
\end{array}$ & $\begin{array}{r}144.29 \\
\pm 249.52 \\
\end{array}$ & $\begin{array}{c}57.14 \\
\pm 22.15 \\
\end{array}$ & $\begin{array}{r}150.00 \\
\pm 247.5 \\
\end{array}$ & $\begin{array}{c}165.71 \\
\pm 154.15\end{array}$ & $\begin{array}{r}117.14 \\
\pm 11.13 \\
\end{array}$ & $\begin{array}{r}71.43 \\
\pm 3.76 \\
\end{array}$ & $\begin{array}{c}51.43 \\
\pm 14.64 \\
\end{array}$ & $\begin{array}{l}54.29 \\
\pm 7.89 \\
\end{array}$ & $\begin{array}{c}54.29 \\
\pm 13.97 \\
\end{array}$ & $\begin{array}{c}93.82 \\
\pm 16.22 \\
\end{array}$ & $<10$ & $10-30$ & $31-100$ & $>100$ \\
\hline $\begin{array}{c}\mathrm{TN} \\
\mu \mathrm{g} / \mathrm{l}\end{array}$ & $\begin{array}{c}620.00 \\
\pm 170\end{array}$ & $\begin{array}{l}542.86 \\
\pm 83.81\end{array}$ & $\begin{array}{c}507.14 \\
\pm 156.60\end{array}$ & $\begin{array}{c}542.86 \\
\pm 127.24\end{array}$ & $\begin{array}{c}450.00 \\
\pm 100\end{array}$ & $\begin{array}{c}707.14 \\
\pm 216.85\end{array}$ & $\begin{array}{c}557.14 \\
\pm 142.68\end{array}$ & $\begin{array}{c}492.86 \\
\pm 151.19\end{array}$ & $\begin{array}{l}435.71 \\
\pm 69.01\end{array}$ & $\begin{array}{l}378.57 \\
\pm 75.59\end{array}$ & $\begin{array}{c}350.00 \\
\pm 0.00\end{array}$ & $\begin{array}{c}507.7 \\
\pm 105.4\end{array}$ & $<350$ & $350-650$ & $651-1500$ & $>1500$ \\
\hline $\begin{array}{c}\text { Chl } a^{3} \\
\mu \mathrm{g} / \mathrm{l}\end{array}$ & $\begin{array}{l}15.33 \\
\pm 1.59\end{array}$ & $\begin{array}{l}24.22 \\
\pm 7.20\end{array}$ & $\begin{array}{l}26.29 \\
\pm 5.06\end{array}$ & \begin{tabular}{|l|}
23.50 \\
\pm 1.48
\end{tabular} & $\begin{array}{l}27.99 \\
\pm 0.86\end{array}$ & $\begin{array}{l}28.26 \\
\pm 0.50\end{array}$ & \begin{tabular}{|l|}
32.07 \\
\pm 1.18
\end{tabular} & $\begin{array}{l}35.62 \\
\pm 3.59\end{array}$ & $\begin{array}{l}19.11 \\
\pm 0.38\end{array}$ & $\begin{array}{c}7.63 \\
\pm 2.24\end{array}$ & $\begin{array}{c}4.28 \\
\pm 0.68\end{array}$ & $\begin{array}{c}22.05 \\
\pm 10.12\end{array}$ & $<3.5$ & $3.5-9$ & $9.1-25.0$ & $>25$ \\
\hline $\begin{array}{l}\text { SD } \\
\mathbf{M}\end{array}$ & $\begin{array}{c}1.07 \\
\pm 0.27\end{array}$ & $\begin{array}{c}0.89 \\
\pm 0.14\end{array}$ & $\begin{array}{c}0.91 \\
\pm 0.16\end{array}$ & $\begin{array}{c}2.36 \\
\pm 0.62\end{array}$ & $\begin{array}{c}1.78 \\
\pm 0.63\end{array}$ & $\begin{array}{c}2.21 \\
\pm 0.32\end{array}$ & $\begin{array}{l}1.49 \\
\pm 0.5\end{array}$ & $\begin{array}{c}0.96 \\
\pm 0.23\end{array}$ & $\begin{array}{c}1.08 \\
\pm 0.14\end{array}$ & $\begin{array}{c}1.24 \pm \\
0.14\end{array}$ & $\begin{array}{c}1.40 \\
\pm 0.28\end{array}$ & $\begin{array}{c}1.40 \\
\pm 0.43\end{array}$ & $>4$ & $4-2$ & $1.9-1$ & $<1$ \\
\hline
\end{tabular}

${ }^{1}$ Water Quality (WQ) and trophic status of the lake were evaluated according to Surface Water Quality Directive (Anonymous, 2016).

${ }^{2}$ The number of repetitions.

${ }^{3}$ Chlorophyll $a$ was measured in two pelagial stations $(n=44)$. 
In the samples taken from Lake Mogan, 58 species from the class Bacillariophyceae were identified, two species from Coscinodiscophyceae, and one from Mediophyceae, totaling 61 species. The composition of the species of diatoms in the lake is given in Table 2.

It was found that Achnanthidium minutissimum, Navicula cryptonella, Ulnaria acus, Encyonopsis microcephala, Rhopalodia gibba and Nitzschia dissipata were dominant in the epiphytic diatom community of the lake. When percentage distribution of the epiphytic diatom species was considered, it was found that A. minutissimum was predominant with an average of $17 \%$, followed by $N$. cryptonella with $8.5 \%$ and $U$. acus with $7.3 \%$ (Table 2).

Table 2. Composition of epiphytic diatom species in the research period in Lake Mogan

\begin{tabular}{l|c}
\hline Species & Composition \% \\
\hline Achnanthidium minutissimum (Kützing) Czarnecki & 17.00 \\
Navicula cryptonella Lange-Bertalot & 8.50 \\
Ulnaria acus (Kützing) Aboal & 7.31 \\
Encyonopsis microcephala (Grunow) Krammer & 5.14 \\
Rhopalodia gibba (Ehrenberg) Otto Müller & 4.15 \\
Nitzschia dissipata (Kützing) Rabenhorst & 3.56 \\
Ulnaria ulna (Nitzsch) Compère & 3.36 \\
Diatoma moniliformis (Kützing) D.M.Williams & 3.36 \\
Rhoicosphenia abbreviata (C.Agardh) Lange-Bertalot & 3.16 \\
Gomphonema brebissonii Kützing & 2.96 \\
Cymbella excisa Kützing & 2.77 \\
Diatoma tenuis C. Agardh & 2.77 \\
Epithemia sorex Kützing & 2.77 \\
Navicula cryptocephala Kützing & 2.37 \\
Fragilaria capucina var. vaucheriae (Kützing) Lange-Bertalot & 2.37 \\
Nitzschia palea (Kützing) W.Smith & 1.98 \\
Amphora copulata (Kützing) Schoeman and R.E.M.Archibald & 1.78 \\
Mastogloia smithii Thwaites ex W. Smith & 1.58 \\
Fragilaria capucina Desmazières & 1.58 \\
Gomphonema olivaceum (Hornemann) Brébisson & 1.58 \\
Cymbella neocistula Krammer & 1.58 \\
Navicula tripunctata (O. F. Müller) Bory & 1.38 \\
Epithemia turgida (Ehrenberg) Kützing & 1.19 \\
Navicula radiosa Kützing & 0.99 \\
Encyonema auerswaldii Rabenhorst & 0.99 \\
Mastogloia elliptica (C. Agardh) Cleve & 0.99 \\
Gomphonema italicum Kützing & 0.79 \\
Nitzschia amphibia Grunow & 0.79 \\
Tryblionella hungarica Grunow Frenguelli & 0.59 \\
Gyrosigma acuminatum (Kützing) Rabenhorst & 0.59 \\
\hline &
\end{tabular}




\begin{tabular}{|c|c|}
\hline Ulnaria capitata (Ehrenberg) Compère & 0.59 \\
\hline Encyonema silesiacum (Bleisch) D.G. Mann & 0.59 \\
\hline Fragilaria famelica (Kützing) Lange-Bertalot & 0.59 \\
\hline Cymbella helvetica Kützing & 0.40 \\
\hline Navicula cincta (Ehrenberg) Ralfs & 0.40 \\
\hline Lindavia comta (Kützing) Nakov, Gullory, Julius, Theriot, Alverson & 0.40 \\
\hline Amphora pediculus (Kützing) Grunow & 0.40 \\
\hline Gomphonema intricatum var. vibrio (Ehrenberg) Cleve & 0.40 \\
\hline Nitzschia sigmoidea (Nitzsch) W. Smith & 0.40 \\
\hline Cymbella compacta Østrup & 0.40 \\
\hline Gomphonema pseudoaugur Lange-Bertalot & 0.40 \\
\hline Cyclotella meneghiniana Kützing & 0.40 \\
\hline Anomoeoneis sphaerophora Pfitzer & 0.40 \\
\hline Cocconeis placentula Ehrenberg & 0.40 \\
\hline Melosira varians C. Agardh & 0.40 \\
\hline Cocconeis pediculus Ehrenberg & 0.40 \\
\hline Navicula gregaria Donkin & 0.40 \\
\hline Craticula cuspidata (Kützing) D.G.Mann & 0.20 \\
\hline Caloneis amphisbaena (Bory) Cleve & 0.20 \\
\hline Eunotia bilunaris (Ehrenberg) Schaarschmidt & 0.20 \\
\hline Campylodiscus bicostatus W.Smith in Roper & 0.20 \\
\hline Cymbella lanceolata (C. Agardh) C. Agardh & 0.20 \\
\hline Nitzschia tryblionella Hantzsch & 0.20 \\
\hline Epithemia adnata (Kützing) Brébisson & 0.20 \\
\hline Fallacia pygmaea (Kützing) Stickle and D.G.Mann & 0.20 \\
\hline Pinnularia brebissonii (Kützing) Rabenhorst & 0.20 \\
\hline Cymatopleura solea (Brébisson) W.Smith & 0.20 \\
\hline Sellaphora pupula (Kützing) Mereschkovsky & 0.20 \\
\hline Surirella brebissonii Krammer and Lange-Bertalot & 0.20 \\
\hline Surirella ovalis Brébisson & 0.20 \\
\hline Pinnularia viridiformis Krammer & 0.20 \\
\hline Number of species & 61 \\
\hline
\end{tabular}

Table 3 shows the ecological evaluation of the diatom community in Lake Mogan. The diatom community in the lake was classified by its $\mathrm{pH}$ as alkaliphilic. Oxygen requirement was found to be high. There was a slight salinity, and in terms of nitrogen uptake, autotrophic nitrogen tolerance was found. The saprobity level was estimated as $\beta$-mesosaprobic and $\alpha-\beta$ mesosaprobic. The trophic condition was found to be eutrophic, as having numerous eutrophication-tolerant species (Table 3). 
Table 3. Estimation of ecological parameters according to epiphytic diatom abundance in Lake Mogan

\begin{tabular}{|c|c|c|c|c|c|c|c|c|c|c|c|}
\hline $\begin{array}{c}\text { Van Dam et al. } \\
\text { (1994) }\end{array}$ & 1 & 2 & 3 & 4 & 5 & 6 & 7 & & & & DOMINANT \\
\hline $\mathrm{pH}$ & 0 & 2 & 253 & 472 & 146 & 0 & & & & & 4 alkaliphilous \\
\hline Salinity & 0 & 753 & 57 & 63 & & & & & & & 2 fresh-brackish water \\
\hline $\begin{array}{l}\text { Nitrogen } \\
\text { uptake }\end{array}$ & 204 & 462 & 18 & 20 & & & & & & & $\begin{array}{c}2 \mathrm{~N} \text {-autotrophic } \\
\text { tolerant }\end{array}$ \\
\hline $\begin{array}{l}\text { Oxygen } \\
\text { requirements }\end{array}$ & 271 & 196 & 200 & 36 & 4 & & & & & & 1 high \\
\hline Saprobity & 67 & 575 & 126 & 40 & 20 & & & & & & $2 \beta$-mesosaprobe \\
\hline Trophic status & 0 & 0 & 26 & 117 & 362 & 20 & 322 & & & & 5 eutrophentic \\
\hline Moisture & 109 & 261 & 443 & 6 & 0 & & & & & & 3 aquatic \\
\hline $\begin{array}{l}\text { Hofmann } \\
\text { (1994) }\end{array}$ & $\mathbf{0}$ & 1 & 2 & 3 & 4 & 5 & 6 & 7 & 8 & 9 & \\
\hline Trophic state & 172 & 0 & 8 & 0 & 103 & 292 & 405 & 0 & 20 & & $\mathbf{6}$ tolerant \\
\hline Sabrobity & 184 & 8 & 123 & 235 & 14 & 289 & 4 & 91 & 34 & 20 & $\begin{array}{l}\mathbf{5} \text { alfa-beta } \\
\text { mesosaprobe }\end{array}$ \\
\hline $\begin{array}{c}\text { Håkansson } \\
\text { (1993) }\end{array}$ & 1 & 2 & 3 & 4 & 5 & 6 & 7 & 8 & 9 & & \\
\hline Classes $\mathrm{pH}$ & 0 & 0 & 2 & 2 & 117 & 180 & 391 & 16 & 40 & & 7 alkaliphilic \\
\hline Denys (1991) & $\mathbf{0}$ & 1 & 2 & 3 & 4 & 5 & 6 & 7 & 8 & & \\
\hline Habitat & 166 & 0 & 45 & 482 & 132 & 174 & 0 & 0 & 0 & & $\begin{array}{c}3 \text { tychoplanktonic, } \\
\text { epontic origin }\end{array}$ \\
\hline Current & 184 & 0 & 0 & 32 & 773 & 12 & & & & & 4 indifferent \\
\hline
\end{tabular}

Results were made of the variations by station of diatom indices in Lake Mogan (Table 4). In calculation of diatom indices, $100 \%$ of the species identified were used for IDG, $97-100 \%$ for IPS, $88-96 \%$ for EPI-D, $81-89 \%$ for IBD, $76-85 \%$ for TDI, $64-67 \%$ for TDIL, 74-85\% for TID, 70-83\% for CEE, 63-73\% for SID, 61-73\% for IDSE, 60$73 \%$ for SLA, $58-70 \%$ for SHE, $53-67 \%$ for DI-CH, $44-57 \%$ for IDP, $38-50 \%$ for DESCY, 35-51\% for LOBO, 27-43\% for IDAP, and 20-33\% for WAT.

Table 4. The variation of diatom indices according to stations in Lake Mogan

\begin{tabular}{c|c|c|c|c|c|c|c}
\hline Station & DESCY & WAT & TDI & IDG & IPS & IDP & IDP \\
& $\mathbf{1 - 5}$ & $\mathbf{0 - 1 0 0}$ & $\mathbf{0 - 1 0 0}$ & $\mathbf{1 - 5}$ & $\mathbf{1 - 5}$ & $\mathbf{0 - 4}$ & \\
\hline 1 & 3.84 & 68.74 & 52.56 & 3.73 & 3.61 & 1.63 & III \\
2 & 3.87 & 71.72 & 48.50 & 3.80 & 3.66 & 1.52 & III \\
3 & 3.96 & 66.85 & 52.5 & 3.68 & 3.42 & 1.68 & III \\
4 & 3.66 & 69.07 & 48.15 & 3.74 & 3.57 & 1.67 & III \\
5 & 3.74 & 68.74 & 52.34 & 3.72 & 3.56 & 1.67 & III \\
\hline
\end{tabular}

The correlation between diatom indices and the physical and chemical parameters measured in the lake was examined, and it was found that most of the diatom indices were correlated with the physical and chemical parameters (Table 5). 
Table 5. Correlations between diatom indices and physical-chemical parameters in Lake Mogan ( $n=55)$

\begin{tabular}{|c|c|c|c|c|c|c|c|c|c|c|c|c|c|c|c|c|c|c|}
\hline Indices & SLA & DESCY & IDSE & SHE & WAT & TDI & $\% \mathrm{PT}$ & IDG & CEE & IPS & IBD & IDAP & EPI-D & DI-CH & IDP & LOBO & SID & TID \\
\hline SD & 0.006 & $-0.379^{* *}$ & 0.005 & $0.277^{*}$ & 0.135 & -0.073 & -0.193 & $0.338^{*}$ & 0.168 & 0.125 & 0.096 & 0.073 & -0.209 & 0.002 & -0.084 & 0.234 & -0.146 & -0.051 \\
\hline Temp & $-0.578^{* *}$ & $-0.347^{* *}$ & $0.521^{* *}$ & $0.798^{* *}$ & $0.466^{* *}$ & $-0.298^{*}$ & $-0.487^{* *}$ & $0.716^{* *}$ & $0.523^{* *}$ & 0.237 & $0.426^{* *}$ & $0.387^{* *}$ & $-0.691^{* *}$ & $-0.468^{* *}$ & $-0.444^{* *}$ & $0.666^{* *}$ & $-0.462^{* *}$ & $-0.411^{* *}$ \\
\hline pH & 0.065 & 0.250 & $0.358^{* *}$ & $0.378^{* *}$ & $-0.388^{* *}$ & $-0.673^{* *}$ & $-0.626^{* *}$ & $0.546^{* *}$ & $0.417^{* *}$ & $0.797^{* *}$ & $0.504^{* *}$ & 0.101 & -0.011 & $-0.540^{* *}$ & $-0.606^{* *}$ & -0.129 & $-0.645^{* *}$ & $-0.422^{* *}$ \\
\hline EC & 0.257 & -0.104 & $-0.347^{* *}$ & -0.195 & $-0.317^{*}$ & 0.121 & $0.311^{*}$ & -0.151 & -0.191 & -0.074 & -0.178 & -0.182 & 0.122 & 0.224 & 0.262 & -0.260 & 0.150 & 0.094 \\
\hline DO & $0.550^{* *}$ & $0.465^{* *}$ & $-0.286^{*}$ & $-0.561^{* *}$ & $-0.561^{* *}$ & 0.061 & 0.132 & $-0.467^{* *}$ & $-0.372^{* *}$ & 0.149 & -0.128 & $-0.379^{* *}$ & $0.633^{* *}$ & 0.163 & 0.090 & $-0.614^{* *}$ & 0.191 & 0.205 \\
\hline TP & -0.170 & -0.027 & 0.040 & 0.148 & $0.409^{* *}$ & 0.112 & -0.124 & 0.100 & 0.141 & -0.051 & 0.068 & 0.068 & -0.223 & -0.120 & 0.021 & 0.208 & 0.043 & 0.072 \\
\hline TN & -0.180 & -0.248 & -0.059 & 0.091 & $0.414^{* *}$ & 0.220 & 0.145 & -0.068 & -0.055 & -0.235 & -0.054 & 0.077 & -0.194 & 0.128 & 0.143 & 0.248 & 0.097 & 0.124 \\
\hline $\mathrm{SiO}_{2}$ & 0.182 & 0.201 & 0.139 & 0.160 & -0.255 & $-0.380^{* *}$ & $-0.568^{* *}$ & $0.302^{*}$ & 0.164 & $0.593^{* *}$ & $0.341^{*}$ & -0.099 & 0.094 & $-0.363^{* * *}$ & $-0.362^{* * *}$ & -0.195 & -0.260 & -0.195 \\
\hline
\end{tabular}

${ }^{1}$ SLA: Sládečék Index, EPI-D: Eutrophication Pollution Index - Diatoms, LOBO: Lobo's Index, TID: Rott's Trophic Index, SID: Rott's Saprobic Index, IDP: Pampean Diatom Index, DESCY: Descy's Index, IDSE: Leclercq and Maquet's Index, TDIL: Trophic Diatom Index for Lakes, SHE: Steinberg and Schiefele's Index, DI-CH: Swiss Diatom Index, IPS: Specific Pollution Index, IDG: Generic Diatom Index, IDAP: Artois-Picardie Diatom Index, IBD: Biological Diatom Index, CEE: European Index, WAT: Diatom Assemblage Index for Organic Pollution, TDI: Trophic Diatom Index, \%PT: \% of Pollution Tolerant Taxa

${ }^{*} \mathrm{p}<0.05^{* *} \mathrm{p}<0.01$ 


\section{Discussion}

In this study on Lake Mogan, the ecological quality of the lake was estimated in relation to epiphytic diatoms and various parameters of water quality. Considering the composition of species identified in the study, it was found that most were alkaliphilic species with high oxygen requirements according to Håkansson (1993) and Van Dam et al. (1994). This is supported by the $\mathrm{pH}$ and oxygen values of the lake. The saprobity level was estimated as $\beta$-mesosaprobic according to Van Dam et al. (1994), and $\alpha-\beta$ mesosaprobic according to Hofmann (1994). In addition, it was found to contain fresh and saltwater species which prefer water rich in electrolytes. Most species were found in $\beta$-mesosaprobic and $\alpha$-mesosaprobic environments in terms of organic pollution, and in terms of trophic level, most species were eutrophic and organic pollution tolerant. The trophic condition was found to be eutrophic according to Van Dam et al. (1994), and according to Hofmann (1994) as having numerous eutrophication-tolerant species.

The mean IBD, EPI-D, DI-CH, SID, CEE, IDAP and IDSE indices calculated according to the epiphytic diatoms identified in the lake indicated that the water quality of the lake was in class II, and the mean TID index showed a water quality of class IIIII. Lake Mogan showed class III ( $\alpha$-mesosaprobic) water quality according to WAT index. Values of 3 and 4 according to DESCY index and of 1.5 and 2.0 according to IDP index indicated medium nutrient-rich waters (Descy, 1979; Gómez and Licursi, 2001). Averages of 3.81 for DESCY index and 1.63 for IDP index were estimated. IDG, IPS, IDP, WAT, DESCY and TDI indices calculated according to epiphytic diatoms indicated class III water quality. According to these values, Lake Mogan is in class III of water quality (critic level pollution and eutrophication). TDIL, developed with the investigation of epiphytic diatoms on $P$. australis in shallow lakes in Hungary, was applied in Lake Mogan, and the proportion of the species used in the index was found to be 64-67\%. The indices used in different countries have been developed for water sources in different typologies. It was determined that the greatest number of species was used by IDG. This is because in this index, taxonomy is at the genus level, and this greatly eases its application to many bodies of water. According to chlorophyll $a$ and total phosphorus concentration, Lake Mogan is eutrophic. The occurrence of eutrophic and pollution-tolerant species and ecological quality varying from medium to poor on most indices indicated an increase in organic material and eutrophication in Lake Mogan.

According to SLA and SHE indices, Lake Mogan showed class I water quality, while according to the average LOBO index, the lake showed class $\mathrm{V}$ water quality. The results of SLA, SHE and LOBO index were not in accordance with water quality parameters and other diatom indices. Therefore, SLA, SHE and LOBO indices are not suitable for use in future water quality monitoring studies in Lake Mogan.

According to the results obtained in this study, it could be said that IDG, IPS, IDP, DESCY, WAT and TDI indices reflected water quality of lake. Various researchers have shown that diatom indices calculated with diatom compositions found in the littoral regions of lakes can be used to reflect their trophic conditions. Blanco et al. (2004) monitored the water quality of six lakes in Spain, and reported that there was a significant correlation between the total nitrogen concentration and IBD and IPS indices, and that this correlation was lower than with phosphorus concentration. Acs et al. (2005) calculated IBD, IPS and IDG indices in monitoring the water quality of Lake Velence in Hungary, and concluded that IBD index could be the most suitable. Lirika et al. (2013) used Trophic Diatom Index (TI DIA $)$ and Saprobic Index (SI) to determine the 
water quality of Lake Ohrid. Kavya and Ulavi (2014) used Louis Leclercq IDSE index in Lakes Karanji and Kukkarahalli in India. Blanco et al. (2004) found IPS suitable in six lakes in Spain, Acs et al. (2005) found IPS and IDG suitable in Lake Velence. In Turkey, Tokatlı (2013) used TDI index in calculating the water quality of Porsuk Reservoir, and reported that the water quality of the lake was mesotrophic.

A significant correlation was found in this study between total phosphorus and total nitrogen concentrations and WAT index. In studies conducted in Turkey on epilithic diatoms in running water, Solak et al. (2007) reported that in Muğla-Akçay (Great Meander river) SLA, DESCY, IDP, LMA, WAT, CEE and IDAP indices showed high correlation with chemical variables. Solak (2009), working on Felent river in Kütahya, and Akbulut et al., (2010) on Kizılırmak river, found that IDAP, WAT, CEE and IPS indices had the highest correlations with physical and chemical parameters. Kivrak et al., (2012) calculated EPI-D, SID, TDI and IDP indices in Akarçay river in Afyonkarahisar, and reported that these four indices, used in Akarçay and sensitive to organic pollution, could be successfully used in evaluating water quality in running waters in Turkey. Gün (2011), used SLA, DESCY, IDSE, SHE, TDI, GENRE, IPS, IBD, EPI-D, DI-CH, IDP, SID and TID indices in the Değirmendere river. It was concluded that the indices which showed the most deviation from physical and chemical parameters were TDI and DESCY. Tokatl (2012) reported the water quality of the Upper Basin of the Gürleyik river as meso-eutrophic according to TDI index, and as mesotrophic according to IBD index.

In Turkey, apart from a study by Tokatl (2013) to determine the water quality of Porsuk Reservoir using Trophic Diatom Index (TDI), no study was found monitoring the water quality of a lake using diatom indices. As a result, IDG and IPS are the most suitable for use in future studies of Lake Mogan. IDG, IPS, IDP, DESCY, WAT and TDI indices reflected water quality of lake but IDG and IPS also integrated all diatom taxa within the samples.

\section{Conclusion}

In conclusion, epiphytic diatoms can be used to monitor ecological quality in shallow lakes such as Lake Mogan, which are rich in aquatic macrophytes and surrounded by reed belts. Lake Mogan is an important example of a shallow lake which is under threat from pollution even though it has a protected status. Biological monitoring studies must continue concerning the sustainable use of the lake, and steps must be taken to prevent its pollution. It is predicted that the research conducted will contribute to the pool of knowledge for the protection and restoration of the lake.

Acknowledgements. This study is part of a doctoral thesis supported by Ankara University Scientific Research Coordination Office, Project No: 13L4347003.

\section{REFERENCES}

[1] Acs, E., Szabo, K., Toth, B., Kiss, K.T. (2004): Investigation of benthic algal communities, especially diatoms of some Hungarian streams in connection with reference conditions of the Water Framework Directives. - Acta Botanica Hungarica 46 (3-4): 255-277.

[2] Acs, E., Rescone, N.M., Szabo, K., Taba, G.Y., Kiss, K.T. (2005): Application of 
Epiphytic Diatoms in Water Quality Monitoring of Lake Velence - Recommendations and Assignments. - Acta Botanica Hungarica 47 (3-4): 211-223.

[3] Akbulut, N., Akbulut, A. (2002): The Plankton Composition of Lake Mogan in Central Anatolia. - Zoology in the Middle East 27: 107-116.

[4] Akbulut, N., Akbulut, A., Günsel, S., Solak, C.N. (2010): Use of epilithic diatoms to evaluate water quality of Kizılırmak River. - Hacettepe University Fund, Final Report No: 0701601006 (In Turkish).

[5] Anonymous (2000): Water Framework Directive, EU. Retrieved from: http://www.ec.europa.eu/environment/water/water-framework/index-en.html

[6] Anonymous (2015): Special Protection Areas. Ministry of Environmental and Urbanism of Turkey. Retrieved from: http://www.csb.gov.tr/gm/tabiat/index.php?Sayfa = sayfa\& Tur= webmenu\&Id=195 (in Turkish).

[7] Anonymous (2016): Surface Water Quality Directive.10.08.2016. - Official Gazette: 2979 (in Turkish).

[8] APHA. (1998): Standart Methods for the Examination of Water and Wastewater $\left(20^{\text {th }}\right.$ ed.) - American Public Health Association, Washington.

[9] Atıcı, T. (1997): Sakarya river pollution and algae. - Ekoloji ve Çevre Dergisi 6 (24): 2832 (in Turkish).

[10] Atıc1, T., Obalı, O., Altındağ, A., Ahıska, S., Aydın, D. (2010): The accumulation of heavy metals $(\mathrm{Cd}, \mathrm{Pb}, \mathrm{Hg}, \mathrm{Cr})$ and their state in phytoplanktonic algae and zooplanktonic organisms in Beysehir Lake and Mogan Lake, Turkey. - African Journal of Biotechnology 9 (4): 475-487.

[11] Atıc1, T., Tokatl, C. (2014): Algal Diversity and Water Quality Assessment with Cluster Analysis of Four Freshwater Lakes (Mogan, Abant, Karagöl and Poyrazlar) of Turkey. Wulfenia 21: 155-169.

[12] Bellinger, E.G., Sigee, D.C. (2010): Freshwater Algae: identification and use as bioindicators. - John Wiley and Sons, UK.

[13] Blanco, S., Ector, L., Bécares, E. (2004): Epiphytic Diatoms as Water Quality Indicators in Spanish Shallow Lakes. - Vie Milieu 54 (2-3): 71-79.

[14] CEN. (2004): Water quality - Guidance standard for the identification, enumeration, and interpretation of benthic diatom samples from rivers. - Comitee Europeen de Normalization, Geneva.

[15] Cox, E.J. (1991): What is the basis for using diatoms as monitors of river quality? - In: Whitton, B.A., Rott, E., Fredrich, G. (eds) Use of Algae for Monitoring Rivers, Universitat Innsbruck, 33-40.

[16] Cox, E.J. (1996): Identification of Freshwater Diatoms from Live Material. - Chapman and Hall, London.

[17] Demir, A.N., Fakığlu, Ö., Dural, B. (2014): Phytoplankton functional groups provide a quality assessment method by the Q assemblage index in Lake Mogan (Turkey). Turkish Journal of Botany 38: 169-179.

[18] Demir, N., Çetin, T., Gök, C., Şanal, M. (2017): First Biological Monitoring in the Akarçay Basin According to the Water Framework Directive: Phytoplankton and Phytobenthos. - Turkish Journal of Water Science \& Management 1(1): 90-107.

[19] Denys, L. (1991): A check-list of the diatoms in the Holocene deposits of the western Belgian coastal plain with a survey of their apparent ecological requirements. -Belgian Geological Survey N 246, Belgium.

[20] Descy, J.P. (1979): A new approach to water quality estimation using diatoms. -Nova Hedwigia 64: 305-323.

[21] Eloranta, P., Soininen, J. (2002): Ecological status of some Finnish rivers evaluated using benthic diatoms. - Journal of Applied Phycology 14: 1-7. 
[22] Fakığlu, Ö., Pulatsü, S. (2005): Determination of External Phosphorus Loading in Mogan Lake (Ankara) Following Some Restoration Measures. -Yüzüncü Y1l Üniversitesi Ziraat Fakültesi Tarım Bilimleri Dergisi 15 (1): 63-69 (in Turkish).

[23] Gómez, N., Licursi, M. (2001): The Pampean Diatom Index (IDP) for assessment of rivers and streams in Argentina. - Aquatic Ecology 35: 173-181.

[24] Guiry, M.D., Guiry, G.M. (2018): Algaebase. Worldwide electronic publication. National University of Ireland, Gelway. Retrieved from http://www.algaebase.org.

[25] Gün, B. (2011): The water quality of Degirmendere Stream determinations by physicochemical parameters and epilithic diatoms and comparisons of the water quality index (MSc Thesis). - Süleyman Demirel Üniversitesi, Fen Bilimleri Enstitüsü, Isparta, Türkiye (in Turkish).

[26] Håkansson, S. (1993): Numerical Methods for the Inference of $\mathrm{pH}$ variations in mesotrophic and eutrophic lakes in Southern Sweden. - A Progress Report Diatom Research 8(2): 349-370.

[27] Hofmann, G. (1994): Aufwuchs diatoms in Seen und ihre Eignung als Indikatoren der Trophie. - Bibliotheca Diatomologica, 30.

[28] Johnson, R. K., Hering, D., Furse, M. T., Clarke, R. T. (2006): Detection of ecological change using multiple organism groups: metrics and uncertainty. - In: Furse, M.T., Hering, D., Brabec, K., Buffagni, A., Sandin, L., Verdonschot, P.F.M. (eds) The Ecological Status of European Rivers: Evaluation and Intercalibration of Assessment Methods. Hydrobiologica 566: 115-137.

[29] Kalyoncu, H., Barlas, M., Ertan, Ö.O. (2009): The study of the water quality of the Aksu Stream according to the biotic index (diatoms and invertebrates) and physico-chemical parameters, the relations of the organisms with the water quality. - Tübav Bilim Dergisi 2 (1): 14-25 (in Turkish).

[30] Kalyoncu, H., Şerbetçi, B. (2013): Applicability of Diatom-Based Water Quality Assessment Indices in Dari Stream, Isparta-Turkey. - International Journal of Environmental, Ecological, Geological and Geophysical Engineering 7 (6): 386-394.

[31] Kavya, S., Ulavi, S. (2014): Bacillariophyceae as Ecological Indicators of water Quality in Two lakes of Mysore. - Universal Journal of Environmental Research and Technology 4 (1): 1-11.

[32] Kesici, T., Kocabaş, Z. (2007): Biyoistatistik. - Ankara Üniversitesi Eczacilık Fakültesi. No: 94. Ankara (in Turkish).

[33] Kivrak, E., Uygun, A., Kalyoncu, H. (2012): Application of Diatom Indices to Assess Water Quality of the Akarçay Stream (Afyonkarahisar, Turkey). - Afyon Kocatepe Üniversitesi Fen Bilimleri Dergisi 12: 27-38 (in Turkish).

[34] King, L., Clarke, G., Bennion, H., Kelly, M., Yallop, M. (2006): Recommendations for sampling littoral diatoms in lakes for ecological status assessments. - Journal of Applied Phycology 18: 15-25.

[35] Kovács, C.S., Buczkó, K., Hájnal, É., Padisák, J. (2007): Epiphytic, littoral diatoms as bioindicators of shallow lake trophic status: Trophic Diatom Index for Lakes (TDIL) developed in Hungary. - Hydrobiologia 589: 141-154.

[36] Krammer, K., Lange-Bertalot, H. (1985): Naviculaceae. - Bibliotheca Diatomologia, Band 9. J. Cramer, Berlin-Stuttgart.

[37] Krammer, K., Lange-Bertalot, H. (1986): Bacillariophyceae. 1. Teil: Naviculaceae. - In: Ettl, H., Gerloff, J., Heynig, H., Mollenhauer, D. (eds) Süsswasser flora von Mitteleuropa, Band 2/1. Gustav Fischer Verlag, Stuttgart.

[38] Krammer, K., Lange-Bertalot, H. (1988): Bacillariophyceae. 2. Teil: Bacillariaceae, Epithemiaceae, Surirellaceae. - In: Ettl, H., Gerloff, J., Heynig, H., Mollenhauer, D. (eds) Süsswasserflora von Mitteleuropa, Band 2/2. VEB. Gustav Fischer Verlag, Jena.

[39] Krammer, K., Lange-Bertalot, H. (1991a): Bacillariophyceae. 3. Teil: Centrales, Fragilariaceae, Eunotiaceae. - In: Ettl, H., Gerloff, J., Heynig, H. and Mollenhauer, D. (eds) Süsswasserflora von Mitteleuropa, Band 2/3. Gustav Fischer Verlag, Stuttgart, Jena. 
[40] Krammer, K., Lange-Bertalot, H. (1991b): Bacillariophyceae. 4. Teil: Achnanthaceae, Kritische Ergänzungen $\mathrm{zu}$ Navicula (Lineolatae) und Gomphonema, Gesamtliteraturverzeichnis Teil 1-4. - In: Ettl, H., Gärtner, G., Gerloff, J., Heynig, H., Mollenhauer, D. (eds) Süsswasserflora von Mitteleuropa, Band 2/4. Gustav Fischer Verlag, Stuttgart, Jena.

[41] Lange-Bertalot, H. (2013): Diatomeen im Süßwasser - Benthos von Mitteleuropa. Koeltz Sci. Books, Germany.

[42] Lecointe, C., Coste, M., Prygiel, J. (1993): Omnidia: software for taxonomy, calculation of diatom indices and inventories management. - Hydrobiologia 269/270: 509-513.

[43] Lirika, K., Alma, I., Magdalena, C., Dashnor, K. (2013): Use of diatom and macrophyte index to evaluate the water quality in Ohrid Lake. - Gazi Ünv. Müh. Mim. Fak. Der. 28(2): 393-400 (in Turkish).

[44] Poulíčková, A., Duchoslav, M., Dokulil, M. (2004): Littoral diatom assemblages as bioindicators of lake trophic status: A case study from perialpine lakes in Austria. Eur. J. Phycol. 39: 143-152.

[45] Round, F.E. (1991): Use of Diatoms for Monitoring Rivers. - In: Whitton B.A., Rott E., Friedrich G. (eds) Use of Algae for Monitoring Rivers, STUDIA, Innsbruck, 25-32.

[46] Salomoni, S.E., Rocha, O., Hermany, G., Lobo, E.A. (2011): Application of water quality biological indices using diatoms as bioindicators in the Gravataí river, RS, Brazil. Brazilian Journal of Biology 71(4): 949-959.

[47] Smol, J. P., Stoermer, E. F. (2010): The Diatoms: Applications for the Environmental and Earth Sciences. - Cambridge University Press, Cambridge.

[48] Solak, C.N. (2009): The determination of pollution in the Felent Creek (Porsuk-Kütahya) by using some aquatic organisms (PhD Thesis). - Dumlupınar Üniversitesi Fen Bilimleri Enstitüsü, Kütahya, Türkiye (in Turkish).

[49] Solak, C.N., Fehér, G., Barlas, M., Pabuçcu, K. (2007): Use of epilithic diatoms to evaluate water quality of Akçay Stream (Büyük Menderes River) in Mugla/Turkey. Arch. Für Hydrobiologie Suppl. 161/3-4, Large Rivers 17 (3-4): 327-338.

[50] Solak, C.N., Acs, E. (2011): Water Quality Monitoring in European and Turkish Rivers Using Diatoms. - Turkish Journal of Fisheries and Aquatic Sciences 11: 329-337.

[51] Strickland, J.D.H., Parssons, T.R. (1972): A Practical Handbook Of Seawater Analysis $\left(2^{\text {nd }}\right.$ Ed). - Bull. Fish. Res. Board. Can., Canada.

[52] Szabo, B., Padisak, J., Selmeczy, G. B., Krienitz, L., Casper, P., Stenger-Kovács, C. (2017): Spatial and temporal patterns of bentic diatom flora in Lake Stechlin, Germany. Turkish Journal of Botany 41 (2): 211-222.

[53] Şanal, M., Köse, B., Coşkun, T., Demir, N.(2015): Estimation of Ecological Quality Using Aquatic Macrophytes in Lake Mogan. - Journal of the Institute of Science and Technology 5(4): 51-55 (in Turkish).

[54] Taş, B., Y1lmaz, Ö., Kurt, I. (2015): Epipelic Diatoms as Indicators of Water Quality in the Lower Part of River Melet (Ordu, Turkey). - Türk Tarım Gida Bilim ve Teknoloji Dergisi 3(7): 610-616 (in Turkish).

[55] Tokatl, C. (2012): Use of some diatom indices for evaluating water quality: sample of Gürleyik stream (Eskişehir). - Dumlupınar Üniv. Fen Bilimleri Enstitüsü Dergisi 29: 2128 (in Turkish).

[56] Tokatl1, C. (2013): Evaulation of Water Quality By Using Trophic Diatom Index: Example of Porsuk Dam Lake. - Journal of Applied Biological Sciences 7 (1): 1-4.

[57] Topçu, A., Pulatsü, S. (2017): Evaluation of some management strategies in eutrophic Mogan lake, Turkey: phosphorus mobility in the sediment-water interface. - Applied Ecology and Environmental Research 15(4):705-717.

[58] Toudjani, A.A., Çelekli, A., Gümüş, E.Y., Kayhan, S., Lekesiz, H.Ö., Çetin, T. (2017): A new diatom index to assess ecological quality of running waters: a case study of water bodies in western Anatolia. - Ann. Limnol. (Int. J. Lim.) 53: 333-343. 
[59] TS EN 15708. (2010): Turkish Standards ICS 13.060.70. Water Quality - Guidance standard fort he surveying, sampling and laboratory analysis of phytobenthos in shallow running water, Ankara.

[60] Van Dam, H., Mertens, A., Sinkeldam, J. (1994): A coded checklist and ecological indicator values of freshwater diatoms from the Netherlands. - Netherlands Journal of Aquatic Ecology 28: 117-133.

[61] Yerli, S. V., Kıvrak, E., Gürbüz, H., Manav, E., Mangıt, F., Türkecan, O. (2012): Phytoplankton Community, Nutrients and Chlorophyll a in Lake Mogan (Turkey); with Comparison Between Current and Old Data. - Turkish Journal of Fisheries and Aquatic Sciences 12: 95-104. 